\title{
Clinical Significance of IL-23 Regulating IL-17A and/or IL-17F Positive Th17 Cells in Chronic Periodontitis
}

\author{
Zhenhua Luo, ${ }^{1,2}$ Hui Wang, ${ }^{2,3}$ Yunlong Wu, ${ }^{2}$ Zheng Sun, ${ }^{1}$ and Yafei $\mathrm{Wu}^{2}$ \\ ${ }^{1}$ Department of Periodontics, School of Stomatology, Capital Medical University, Beijing 100050, China \\ ${ }^{2}$ State Key Laboratory of Oral Diseases, West China College of Stomatology, Sichuan University, Chengdu 610041, China \\ ${ }^{3}$ Department of Oral Medicine, School of Stomatology, Capital Medical University, Beijing 100050, China \\ Correspondence should be addressed to Hui Wang; wh3809@163.com
}

Received 13 August 2014; Accepted 5 October 2014; Published 30 November 2014

Academic Editor: Sack Ulrich

Copyright (c) 2014 Zhenhua Luo et al. This is an open access article distributed under the Creative Commons Attribution License, which permits unrestricted use, distribution, and reproduction in any medium, provided the original work is properly cited.

Objective. To investigate the expression level and clinical significance of (IL-17A ${ }^{+}$and/or IL-17F $\mathrm{F}^{+}$Th17 cells under IL-23 regulation in patients of chronic periodontitis (CP) and healthy controls (HC). Materials and Methods. The whole peripheral blood samples were collected from $30 \mathrm{CP}$ patients and 25 healthy controls. Flow cytometry was used to test the (IL-17A ${ }^{+}$and/or IL-17F ${ }^{+}$) Th17 expression level. Recombinant human IL-23 (rhIL-23) was used to detect Th17 differentiation and expansion. Correlation coefficient analysis between Th17 expression level and clinical parameters was analyzed by SPSS software. Results. Flow cytometry results

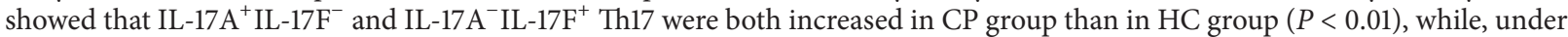
recombinant human IL-23 (rhIL-23) stimulation, the number of IL-17 $\mathrm{A}^{+}$IL-17F $\mathrm{F}^{-}$Th17 cells was significantly increased in both CP and $\mathrm{HC}$ groups $(P<0.01)$. Interestingly, IL-17A $\mathrm{AL}^{-}-17 \mathrm{~F}^{+}$Th17 cells were only increased in CP group after rhIL-23 stimulation. Additionally, correlation coefficient analysis showed significant correlation between IL-17A $\mathrm{A}^{+} \mathrm{IL}-17 \mathrm{~F}^{-}$Th17 cell and attachment loss or probing depth $(P<0.05)$. Conclusions. This study indicates that both the IL-17A $\mathrm{IL}^{+}-17 \mathrm{~F}^{-}$and IL-17A $\mathrm{AL}^{-}-17 \mathrm{~F}^{+}$Th17 cells may be involved in pathogenesis of periodontitis. The role of these Th17 cells in the disease pathogenesis needs to be further investigated.

\section{Introduction}

Periodontitis is the most popular oral chronic inflammatory disease, characterized by the destruction of periodontal tissues around the teeth. In chronic periodontitis patients, cytokines produced by immunocytes can regulate the immune responses to distinct periodontal bacteria like actinobacillus actinomycetemcomitans or porphyromanas gingivalis, and further to play the protective or devastating role in disease progression $[1,2]$. Though the immune mechanism in periodontitis pathogenesis was still not clear, inflammation mediated by $\mathrm{CD} 4^{+} \mathrm{T}$ lymphocytes was already regarded as an important feature of the pathogenesis $[3,4]$.

Th17, the novel population of $\mathrm{CD}^{+}{ }^{+} \mathrm{T}$ cell subsets, has been found more recently for its unique ability to produce interleukin-17 (IL-17, also called IL-17A) [5]. The major role of Th17 cells and their effective cytokine IL-17 has now been described in various models of inflammation mediated destruction and autoimmune diseases, like Crohn's disease or rheumatoid arthritis [6]. Furthermore, previous reports also showed that Th17 cell subset may play a novel important role in pathogenesis of cell-mediated tissue damage caused by immune responses against microbial infection [7]. However, other investigations showed that Th17 cells may produce IL$17 \mathrm{~A}$ to play protective role by neutrophils recruitment to destructed area in periodontitis lesions [8]. Though study on effect of Th17 cells in periodontitis pathogenesis recently attracted much more attention, the specific role of Th17 cell in periodontitis is still disputed now $[9,10]$.

IL-17F recently has drawn much more attention due to its great similarity to IL-17A and can be secreted as homodimers or heterodimers with IL-17A [11]. The genes encoding IL$17 \mathrm{~A}$ and IL-17F are localized in the same chromosomal region and are coexpressed by Th17 cells [12]. Similar to IL17A, IL-17F can also utilize IL-17RA and IL-17RC receptor complex as its signal transducers to induce the expression of proinflammatory cytokines in many human cell types [13]. IL-23 is a newly discovered cytokine and played great 
TABLE 1: Demographic characteristics and clinical parameters in HC and CP groups.

\begin{tabular}{|c|c|c|c|c|c|c|c|}
\hline & \multirow{2}{*}{ Age $^{\mathrm{a}}$ (years) } & \multicolumn{2}{|c|}{ Gender $^{\mathrm{b}}$} & \multirow{2}{*}{$\mathrm{PD}^{\mathrm{a}}(\mathrm{mm})$} & \multirow{2}{*}{$\mathrm{AL}^{\mathrm{a}}(\mathrm{mm})$} & \multirow{2}{*}{$\mathrm{PI}^{\mathrm{a}}$} & \multirow{2}{*}{$\mathrm{GI}^{\mathrm{a}}$} \\
\hline & & Male & Female & & & & \\
\hline $\mathrm{HC}$ group $($ mean $\pm \mathrm{SD})$ & $38.5 \pm 10.2$ & $12(48 \%)$ & $13(52 \%)$ & $1.7 \pm 0.9$ & $0.1 \pm 0.1$ & $0.2 \pm 0.3$ & $0.5 \pm 0.4$ \\
\hline $\mathrm{CP}$ group $($ mean $\pm \mathrm{SD})$ & $42.4 \pm 11.0$ & $15(50 \%)$ & $15(50 \%)$ & $4.5 \pm 1.5$ & $1.4 \pm 0.9$ & $1.1 \pm 0.5$ & $1.2 \pm 0.6$ \\
\hline$P$ value & NS & NS & NS & $P<0.001$ & $P<0.001$ & $P<0.001$ & $P<0.001$ \\
\hline
\end{tabular}

${ }^{\mathrm{a}}$ Student's $t$-test; ${ }^{\mathrm{b}}$ chi-square test.

HC group: healthy controls; CP group: chronic periodontitis; SD: standard deviation; PD: probing depth; AL: attachment loss; PI: plaque index; GI: gingival index; NS represents no statistically significant difference $(P>0.05)$.

important role in differentiation and proliferation of Th17 cells $[14,15]$. Moreover, IL-23 was also found to be involved in human diseases including periodontal diseases. For example, the previous studies demonstrated that both IL-23 mRNA and protein were elevated significantly in periodontal lesions than in control sites $[16,17]$. However, we still do not know whether increased cytokine IL-23 in periodontitis would influence the Thl7 expansion and differentiation.

In our previous studies of periodontitis pathogenesis, we found that serum IL-17A level was elevated in periodontitis patients than healthy controls [18]. However, to the extent of our knowledge, there are still less reports focused on the correlation between (IL-17A ${ }^{+}$and/or IL-17F ${ }^{+}$) Th17 cells and periodontitis. So, we did not know whether (IL-17 $\mathrm{A}^{+}$and/or IL-17F $\mathrm{F}^{+}$) Th17 cells may participate in the disease pathogenesis. Therefore, the present clinical study was designed to detect the expression levels of (IL-17A ${ }^{+}$and/or IL-17F ${ }^{+}$) Th17 cells in periodontitis patients. Furthermore, we would also investigate, under recombinant IL-23 (rhIL23) stimulation, whether more (IL- $17 \mathrm{~A}^{+}$and/or IL-17F ${ }^{+}$) Th17 cells would be produced in disease pathogenesis. And are there any significant correlations between expression levels of (IL-17A ${ }^{+}$and/or IL-17F ${ }^{+}$) Thl7 cells and periodontal clinical parameters?

\section{Subjects and Methods}

2.1. Study Samples. This study was approved by the Institutional Review Board of West China College of Stomatology in Sichuan University (Protocol number S2011108), with informed consent by participants in this study. Careful periodontal examinations were performed at 6 sites per tooth (mesiobuccal, midbuccal, and distobuccal; mesiolingual, midlingual, and distolingual) to record the parameters including clinical attachment loss (AL), probing depth (PD), Silness-Löe plaque index (PI), and Löe-Silness gingival index (GI) scores. The clinical attachment loss (AL) was measured with the distance from depth of periodontal pocket to the cementoenamel junction. The measurement of probing depth (PD) was determined by measuring distance from a gingival margin to the base of the periodontal pocket with a calibrated periodontal probe. All CP patients were enrolled from our Department of Periodontics, with clinically diagnosed and confirmed as chronic periodontitis disease (with pathological attachment loss and probing depth more than $3 \mathrm{~mm}$ ) by the periodontists. Demographic characteristics and clinical parameters of both groups were listed in Table 1. After careful examination, the whole blood sample was drawn for the peripheral blood mononuclear cell (PBMC) extraction.

\subsection{Isolation and Identification of $C D 4^{+} T$ Lymphocytes.} The peripheral blood mononuclear cell (PBMC) extraction was performed by lymphocytes separation kit (Histopaque 10771, Sigma, USA). Separated PBMC cells from human peripheral blood were labeled with the anti-human $\mathrm{CD} 4^{+}$ magnetic particles (Cat. number 557767, BD Bioscience, San Diego, CA, USA). Human $\mathrm{CD}^{+}{ }^{+} \mathrm{T}$ lymphocytes were magnetically separated from $\mathrm{PBMC}$ on the BD IMagnet (Cat. number 552311, BD Bioscience) according to the instruction protocol. The purity of $\mathrm{CD} 4^{+} \mathrm{T}$ cells should be more than 95\% before next step test. To demonstrate the efficiency of the enrichment and selection steps, cells were stained with APC mouse anti-human CD4 (Cat. number 555349, BD Bioscience) and FITC mouse anti-human CD3 (Cat. number 555332, BD Bioscience). Isotype control was stained by Simultest IgG2a/IgG1 (Cat. number 340394, BD Bioscience). Flow cytometry was performed on a Beckman Coulter FC500 flow cytometry system.

2.3. Culture and Stimulation of $C D 4^{+}$T Lymphocytes. $\mathrm{CD} 4^{+}$ $\mathrm{T}$ cell subsets were cultured in RPMI 1640 media containing $10 \%$ FBS (Gibco, USA), $50 \mathrm{U} / \mathrm{mL}$ penicillin, $50 \mu \mathrm{g} / \mathrm{mL}$ streptomycin, with $1 \times 10^{6}$ cells $/ \mathrm{mL}$, in $37^{\circ} \mathrm{C}, 5 \% \mathrm{CO}_{2}$ condition. $\mathrm{CD}^{+} \mathrm{T}$ cells isolated from PBMC were enriched with $2 \mu \mathrm{g} / \mathrm{mL}$ anti-CD3 (Cat. number 555336, BD Bioscience) and $2 \mu \mathrm{g} / \mathrm{mL}$ anti-CD28 (Cat. number 555725, BD Bioscience) monoclonal antibody and $50 \mathrm{ng} / \mathrm{mL}$ recombinant human IL-2 (Cat. number 554603, BD Bioscience). $\mathrm{CD}^{+} \mathrm{T}$ cells were then stimulated as described above and subsequently subjected to $\mathrm{CD} 4^{+}$magnetic separation to increase the purity to $>95 \%$. Human recombinant IL-23 (rhIL-23) was added into the supernatant of $\mathrm{CD}^{+} \mathrm{T}$ cells with a final concentration of $20 \mathrm{ng} / \mathrm{mL}$ to stimulate the $\mathrm{CD}^{+}$differentiation and expansion for 24 hours. Each sample was divided into two groups according to receiving rhIL-23 stimulation or not.

\subsection{Flow Cytometry Detection of $I L-17 A^{+}$and/or $I L-17 F^{+}$} Th17 Cells. After stimulation with rhIL-23 for 24 hours, cells were cultured in the presence of $50 \mathrm{ng} / \mathrm{mL}$ phorbol 12 myristate 13-acetate (P1585, Sigma St. Louis, MO, USA) and $500 \mathrm{ng} / \mathrm{mL}$ ionomycin (I0634, Sigma, USA) and with the protein transport inhibitor BD Golgistop $0.7 \mu \mathrm{L} / \mathrm{mL}$ (Cat. number 554714, BD Bioscience) to prevent the cytokine 


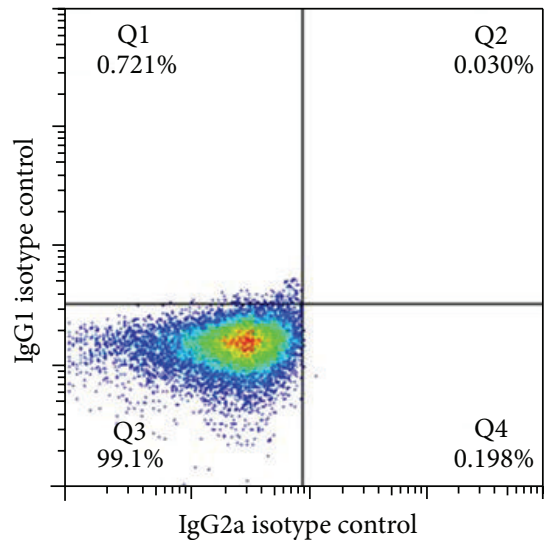

(a)

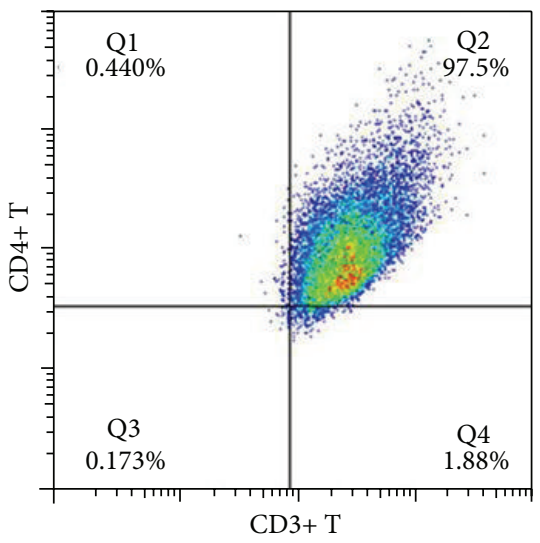

(b)

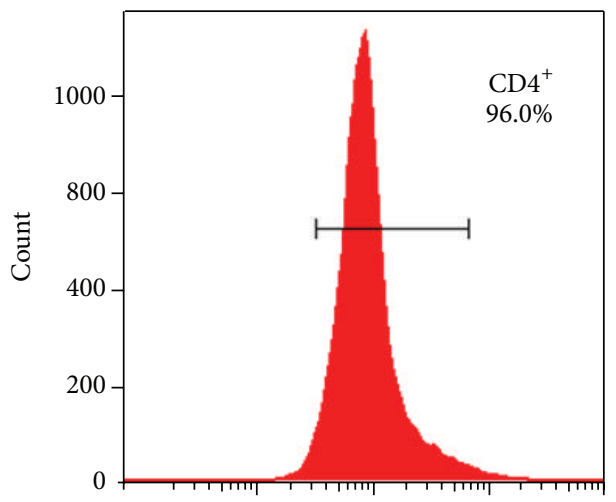

(c)

FIGURE 1: Purity verification of separated $\mathrm{CD} 4^{+} \mathrm{T}$ cells by flow cytometry. (a) showed the isotype control performed by Simultest IgG2a/IgG1. (b) represents double staining by anti-human CD3 and CD4 antibody. (c) represents anti-human antibody CD4 staining. These results showed that the purity of $\mathrm{CD} 4^{+}$from $\mathrm{PBMC}$ can reach a percent of more than $95 \%$.

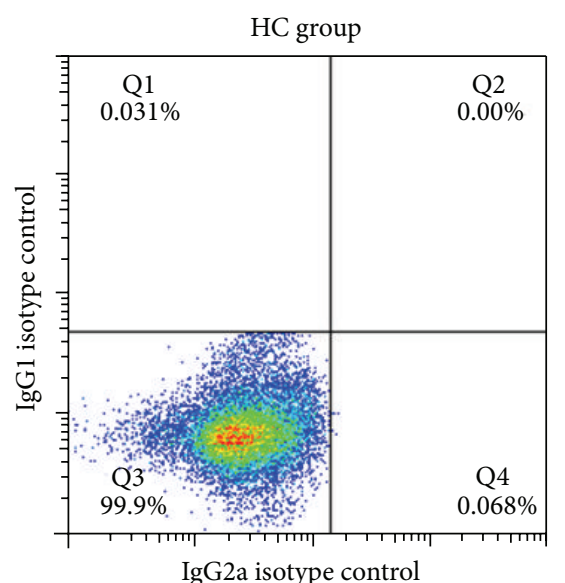

(a)

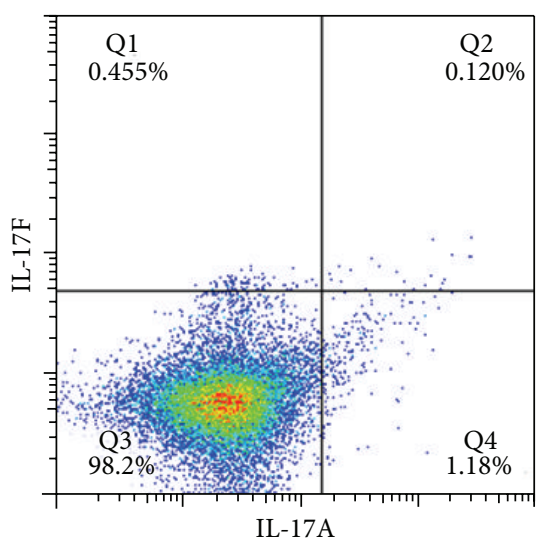

(c)

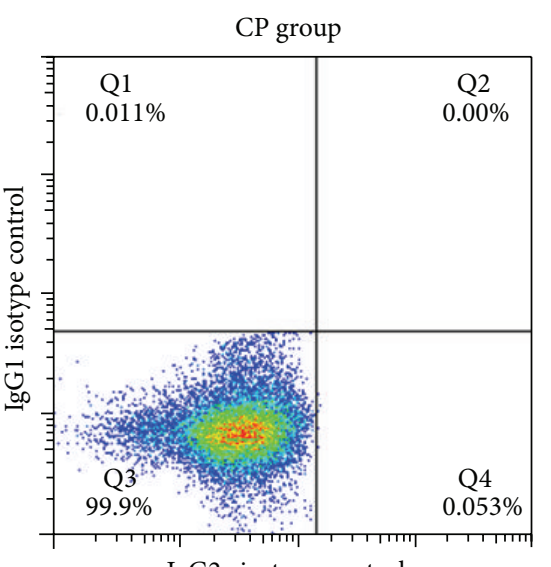

IgG2a isotype control

(b)

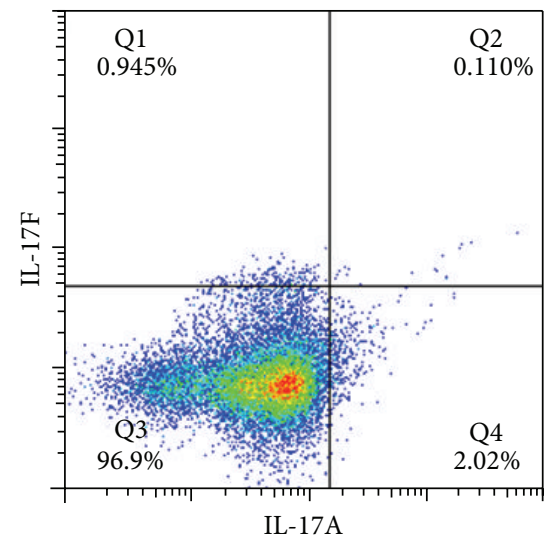

(d)

FIGURE 2: Representative dot blot showing percent expression level of (IL-17A ${ }^{+}$and/or IL-17F $\left.{ }^{+}\right)$Th17 cells in CP group ( $\left.n=30\right)$ and HC group $(n=25)$ within the $\mathrm{CD}^{+}$population. (a) and (b) showed the isotype control performed by Simultest IgG2a/IgG1, while (c) and (d) showed the double staining performed by IL-17A and IL-17F anti-human antibody. 


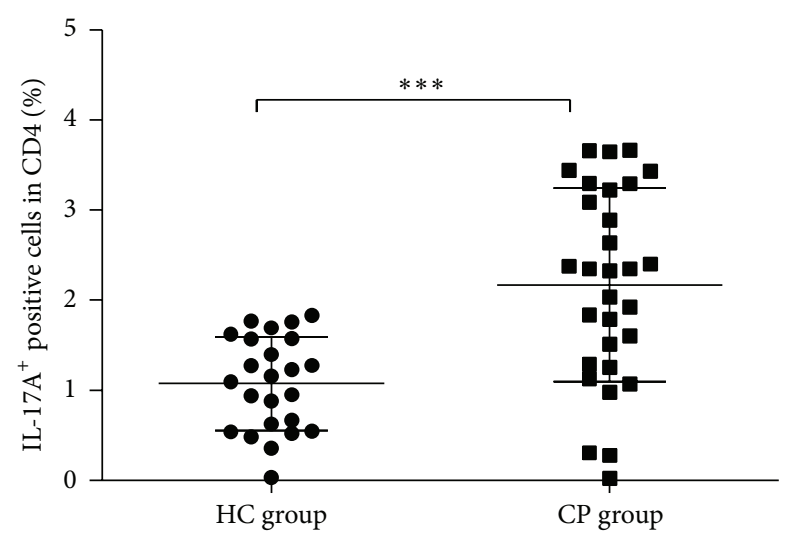

(a)

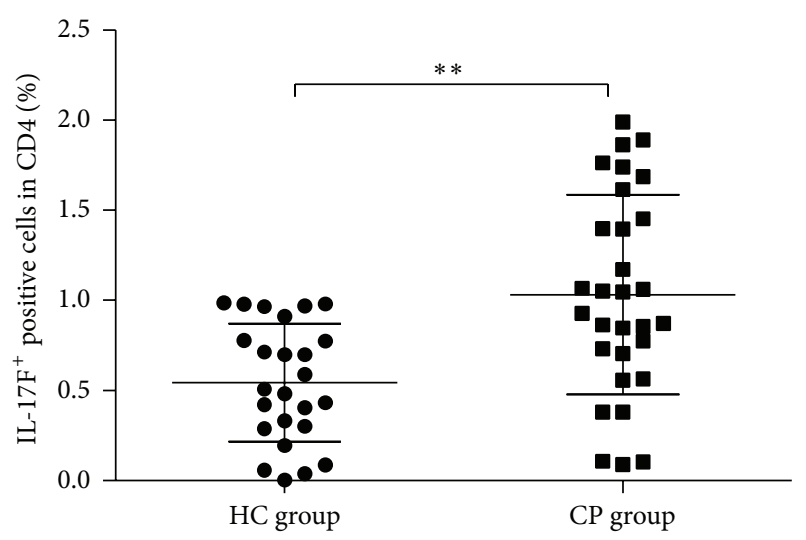

(b)

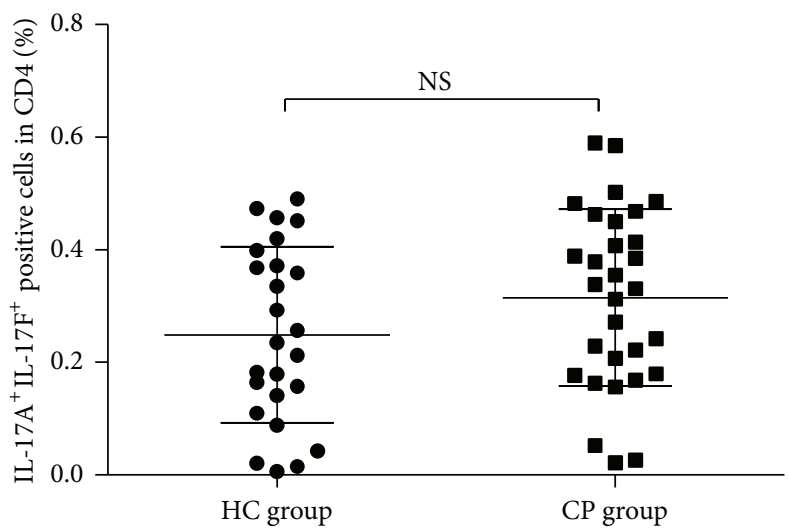

(c)

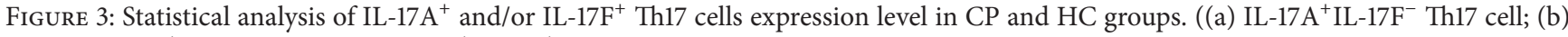
IL-17A IL-17F ${ }^{+}$Th17 cell; and (c) IL-17A ${ }^{+}$IL-17F ${ }^{+}$Th17 cell.)

secretion and allow cytokine accumulation inside the cell. Then, $\mathrm{CD}^{+} \mathrm{T}$ cells were cultured under condition of $37^{\circ} \mathrm{C}$, $5 \% \mathrm{CO}_{2}$, to incubate for 4 hours. Cells were fixed and permeabilized with BD Cytofix/Cytoperm Wash Fixation Solution (Cat. number 554715, BD Bioscience) to allow fluorescent antibodies to enter to bind the targeted cytokines. Using BD Cytofix/Cytoperm (Cat. number 554715, BD Bioscience) to perform the cell fixation and wash buffer. Cells were stained with PE Mouse anti-Human IL-17A (Cat. number 560436, BD Bioscience) and Alexa Fluor 488 Mouse anti-Human IL$17 \mathrm{~F}$ (Cat. number 561331, BD Bioscience). Isotype control was stained with Simultest IgG2a/IgG1 (Cat. number 340394, BD Bioscience). Flow cytometry was performed on a Beckman Coulter FC500 flow cytometry system.

2.5. Statistical Analysis. All data were analyzed by Statistical Package for Social Sciences version 14.0 software (SPSS Inc., Chicago, IL, USA). The chi-square test was used to compare differences in gender in Table 1 . Student's $t$-test was used to compare the means of two samples with both groups. Correlation coefficients were carried out with Spearman's correlation coefficient analysis, where $P<0.05$ was considered to be statistically significant.

\section{Results}

3.1. Expression Level of $I L-17 A^{+} I L-17 F^{-}$and/or $I L-17 A^{-} I L-17 F^{+}$ Th17 Cells in CP and HC Groups. Separated CD $4^{+} \mathrm{T}$ cells were validated by anti-human CD3 and CD4 flow cytometry antibody to test CD4 cell purity. The results showed that the purity of $\mathrm{CD}^{+} \mathrm{T}$ from PBMC can reach a percent of more than 95\% (Figure 1). Flow cytometry of the sample staining with IL-17A and IL-17F antibody was demonstrated in Figure 2 and further data analysis was shown in Figure 3. Flow cytometry analysis showed significantly elevated levels of IL-17A ${ }^{+}$IL-17F ${ }^{-}$Th17 cell (Figure $3(\mathrm{a}), P<0.01$ ) and IL$17 \mathrm{~A}^{-} \mathrm{IL}-17 \mathrm{~F}^{+}$Th17 cell in CP group compared with HC group (Figure 3(a), $P<0.01$ ). However, it did not demonstrate statistically significant difference between $\mathrm{CP}$ group and $\mathrm{HC}$ group with the IL-17A $\mathrm{A}^{+} \mathrm{IL}-17 \mathrm{~F}^{+}$double positive Th17 cell (Figure 3(a), $P>0.05$ ).

3.2. Expression Level of $I L-17 A^{+} I L-17 F$ and/or $I L-17 A^{-} I L-$ $17 F^{+}$Th17 cells in CP and HC Groups under rhIL-23 Stimulation. $\mathrm{CD}^{+} \mathrm{T}$ cells were separated from $\mathrm{CP}$ patients and $\mathrm{HC}$ group peripheral blood with $\mathrm{CD} 4^{+}$magnetic separation kit to increase the purity and were cultured under rhIL-23 stimulation for 24 hours in vitro. Flow cytometry analysis was 


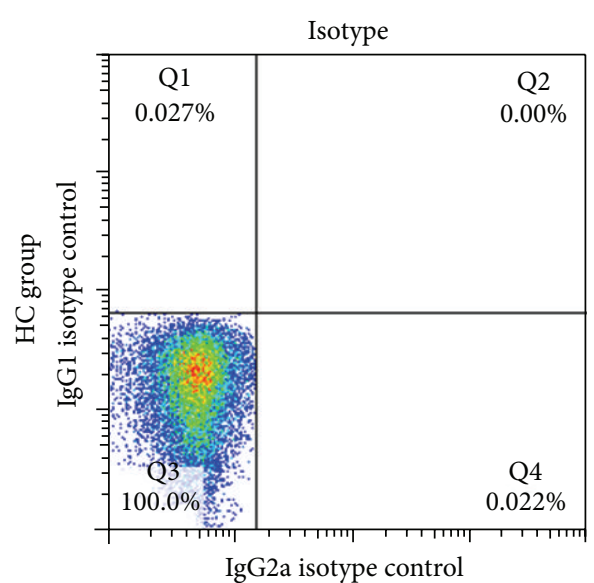

(a)

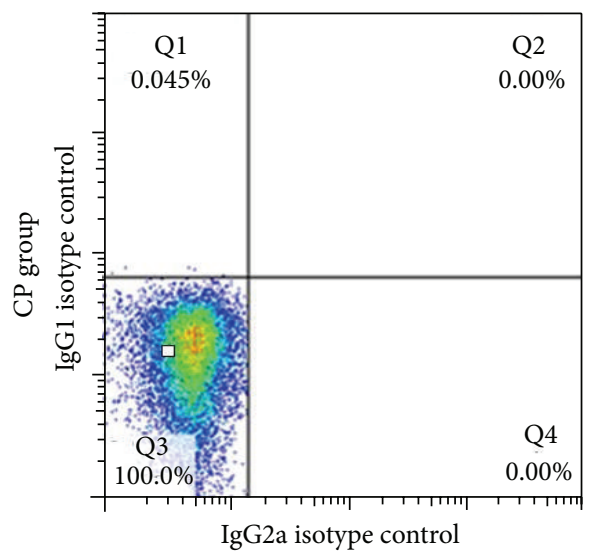

(d)

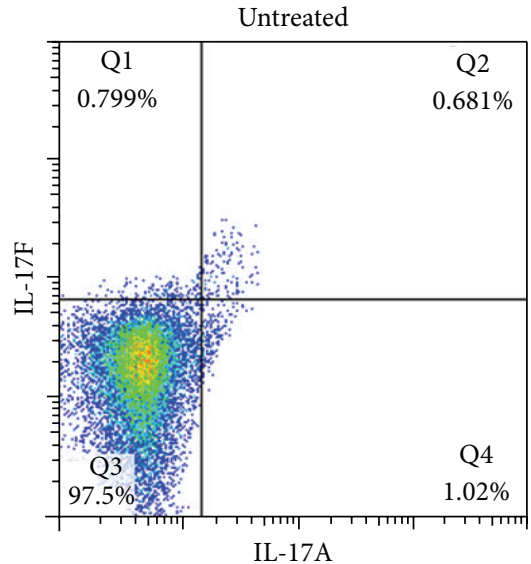

(b)

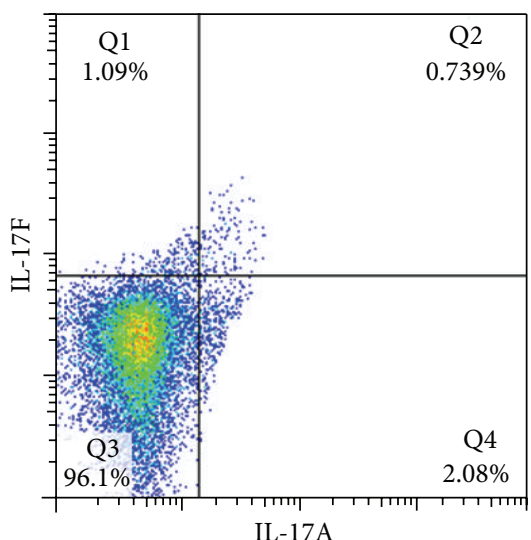

(e)

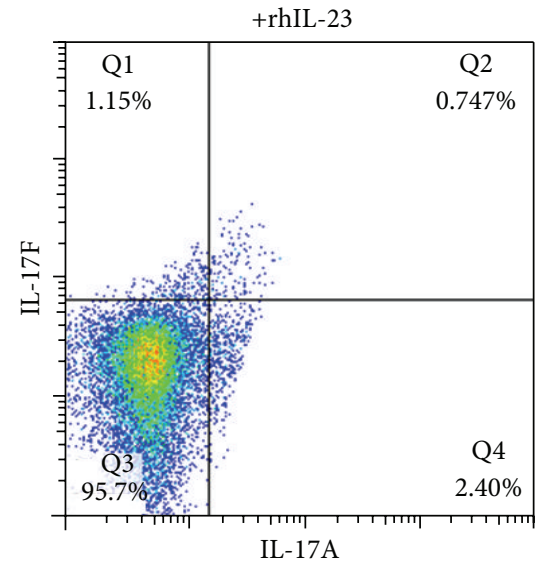

(c)

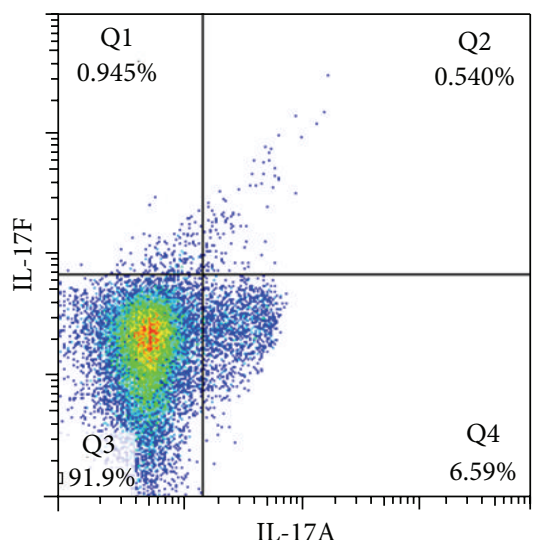

(f)

Figure 4: Expression level of (IL-17A $\mathrm{A}^{+}$and/or IL-17F ${ }^{+}$) Th17 cells in CP and HC groups with or without recombinant IL-23 stimulation. (a) and (d) showed the isotype control performed by Simultest IgG2a/IgG1, while (b), (c), (d), and (e) showed the double staining performed by IL-17A and IL-17F anti-human antibody ((c) and (f) groups were treated by rhIL-23, while (b) and (e) were not). HC group includes (a), (b), and (c); CP group includes (d), (e), and (f).

used to test the percent change in several Th17 cells (Figure 4). It showed a significantly elevated level of IL-17 $\mathrm{A}^{+} \mathrm{IL}-17 \mathrm{~F}^{-}$Th17 cell (Figure 5(a), $P<0.01$ ) in CP group and HC group after rhIL-23 stimulation. However, there is only IL-17 $\mathrm{A}^{-} \mathrm{IL}-17 \mathrm{~F}^{+}$ Th17 cell number significantly increased in $\mathrm{CP}$ group after rhIL-23 simulation (Figure 5(b), $P<0.05$ ), not in HC group (Figure 5(c), $P>0.05)$. Considering IL-17A ${ }^{+}$IL-17F ${ }^{+}$Th17 cell number change after rhIL-23 stimulation, no statistical significance was to be found in $\mathrm{CP}$ group and HC group after rhIL-23 stimulation (Figure 5(c), $P>0.05$ ).

IL-17A ${ }^{+}$IL-17F $F^{-}$and IL-17A ${ }^{-}$IL-17F ${ }^{+}$Th17 cell number were plotted as a function of attachment loss for the $\mathrm{CP}$ patients (Figure 6). It showed obviously that $\mathrm{CP}$ patients demonstrated increased IL-17A $\mathrm{A}^{+} \mathrm{IL}-17 \mathrm{~F}^{-}$and IL-17A $\mathrm{A}^{-} \mathrm{IL}-17 \mathrm{~F}^{+}$ Th17 cells compared with levels in healthy individuals.

3.3. Correlation Coefficients of $I L-17 A^{+} I L-17 F$ and/or $I L-$ $17 A^{-} I L-17 F^{+}$Th17 Cells Expression Level with Clinical Parameters. The correlation coefficient analysis showed positively significant correlations of IL-17A ${ }^{+}$IL- $17 \mathrm{~F}^{-}$Th17 cells with attachment loss and probing depth (Table 2, $P<0.05$ ). It also showed a significant correlation between IL-17A ${ }^{-}$IL$17 \mathrm{~F}^{+}$Th17 cells and attachment loss in CP group (Table 2, $P<0.05)$. However, there was no significant correlation of IL-17A ${ }^{-}$IL-17F $\mathrm{F}^{+}$Th17 cell number and probing depth of CP patients (Table 2, $P>0.05$ ). Additionally, it also showed no significant correlation of two Th17 cells and plaque index or gingival index (Table 2, $P>0.05$ ).

\section{Discussion}

In recent years, Th17 cell has attracted more and more attention of researchers and was identified to be present in peripheral blood and local lesions of periodontitis patients $[19,20]$. Though our previous studies have focused on the expression level of Th17 associated cytokines in periodontitis patients, we still do not know the expression level of IL-17A $\mathrm{A}^{+} \mathrm{IL}-17 \mathrm{~F}^{-}$ and/or IL-17A ${ }^{-}$IL- $17 \mathrm{~F}^{+}$Thl7 cells in CP patients compared to healthy controls. Therefore, this is the first study reporting

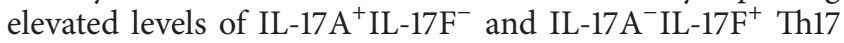




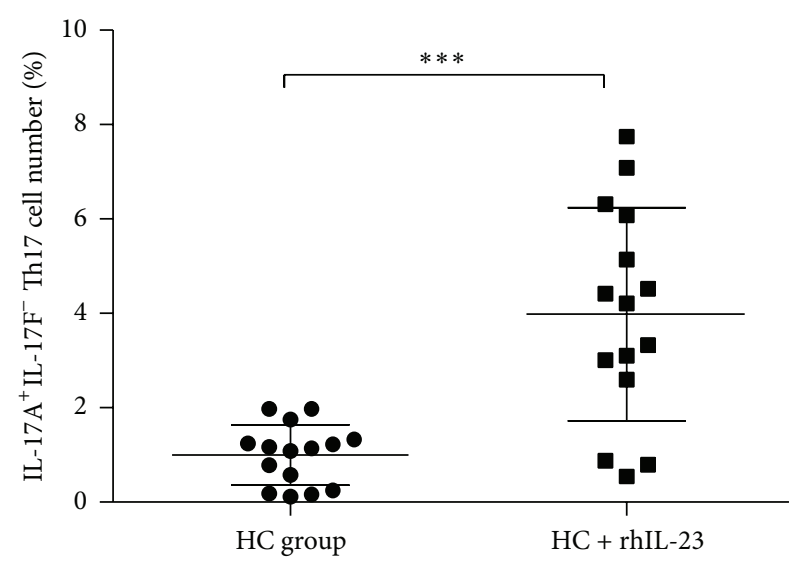

(a)

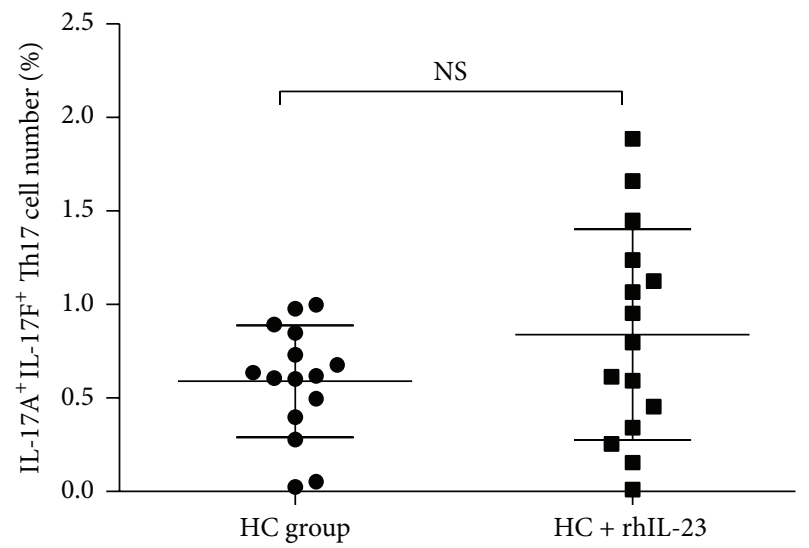

(c)

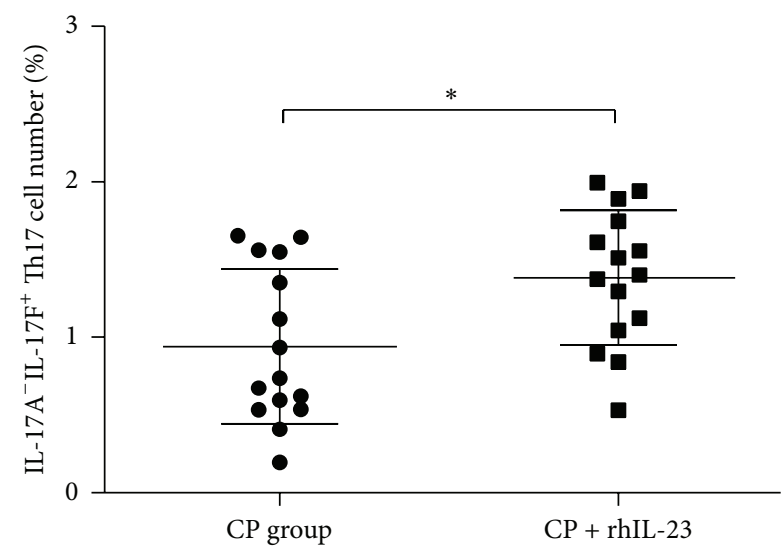

(e)

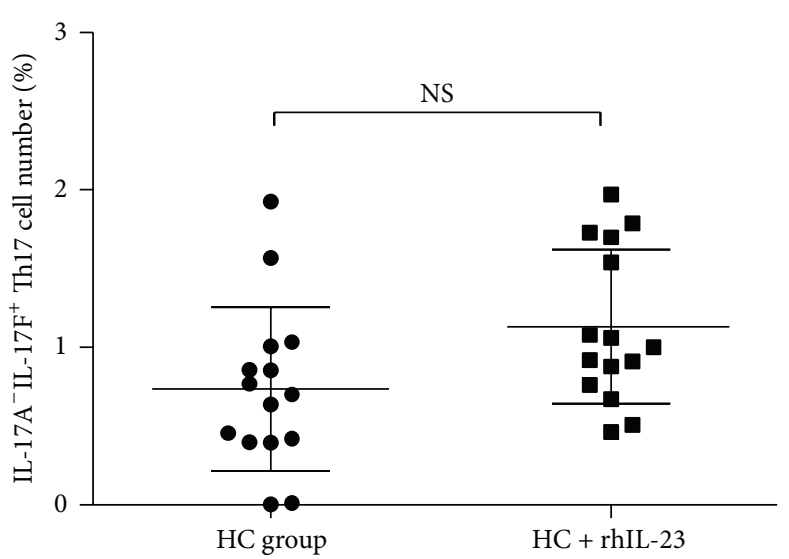

(b)

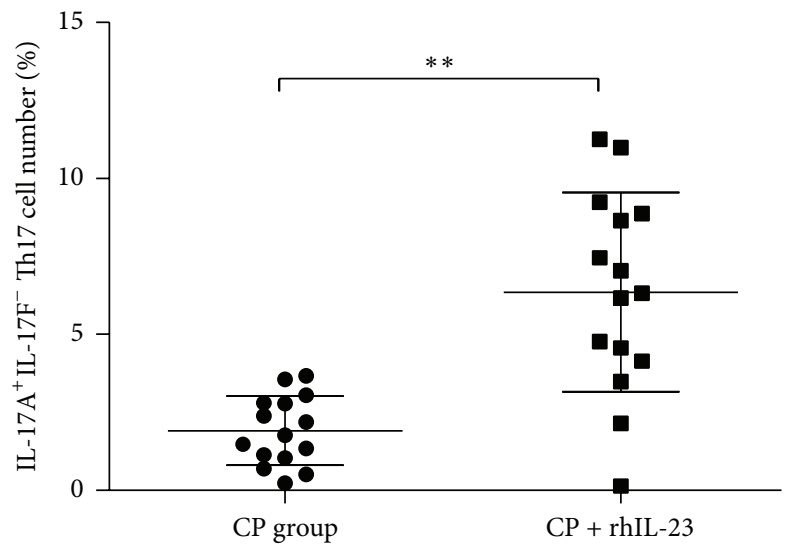

(d)

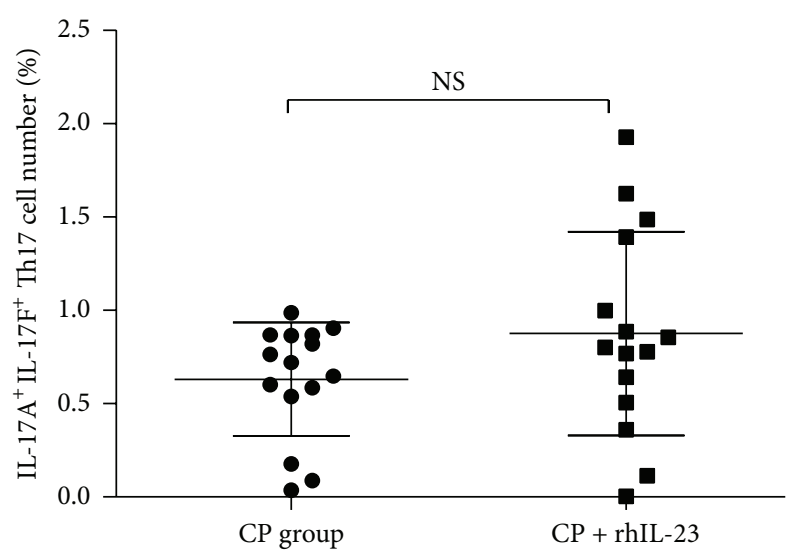

(f)

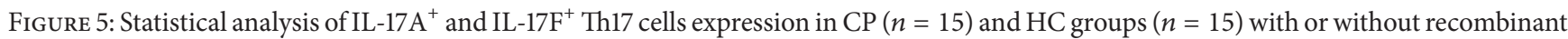

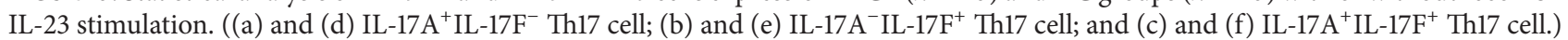

cells in chronic periodontitis. The present results have laid the basis to understand potential role of IL-17 $\mathrm{A}^{-} \mathrm{IL}-17 \mathrm{~F}^{+}$Th17 in the pathogenesis of periodontitis.

Previous in vitro test supports the hypothesis that Th1 cell was associated with periodontal stable lesions, while Th 2 cells with disease progression [21]. For example, previous reports showed that a destructive role for distinct Th cells throughout periodontitis refers to the Th2 subset, which also presented in periodontal lesions [22]. However, besides Th1 and Th2 cells, recent investigations also demonstrated that the Th17 subset may play osteoclastogenic effect [23]. Th17 cells developed through cytokine signals distinct from and antagonized by products from Th1 and Th2 subsets $[24,25]$. Reports showed that Th17 was involved in periodontal bone destruction via 


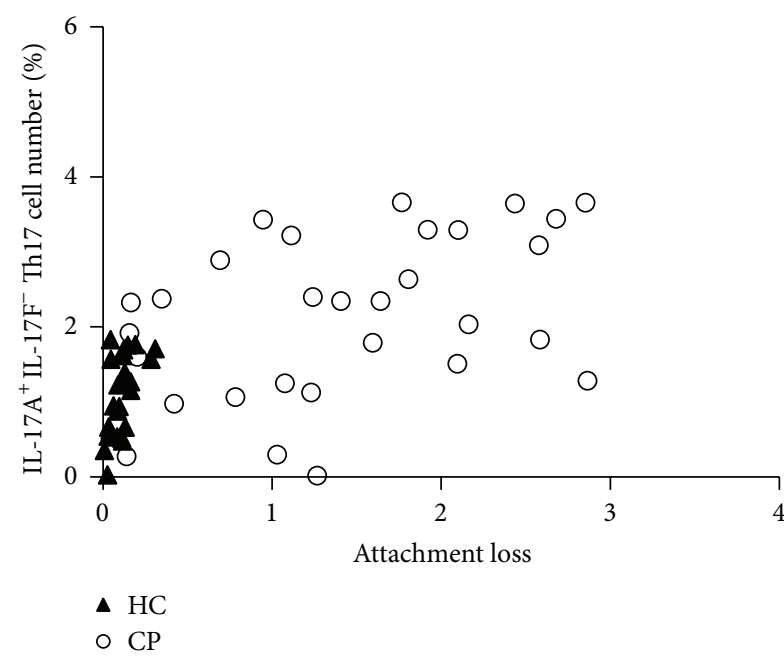

(a)

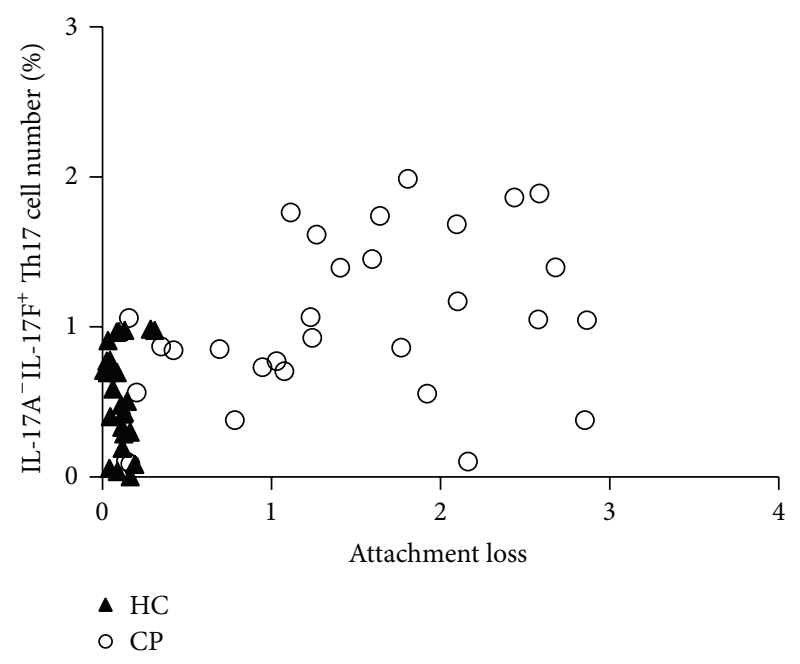

(b)

FIgUre 6: Plot of IL-17A $\mathrm{AL}^{+} \mathrm{IL}-17 \mathrm{~F}^{-}$and $\mathrm{IL}-17 \mathrm{~A}^{-} \mathrm{IL}-17 \mathrm{~F}^{+}$Th17 cells number as a function of attachment loss in chronic periodontitis. (a)

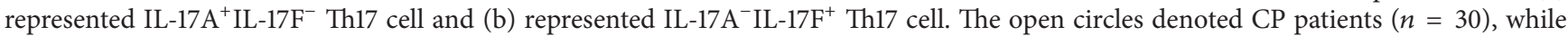
closed triangles denoted periodontally healthy control individuals $(n=25)$.

TABLE 2: Correlation coefficients ( $R$ squared) between two distinct Th17 cells (IL-17A ${ }^{+}$IL-17F ${ }^{-}$and IL-17A ${ }^{-}$IL-17F ${ }^{+}$Th17) expression level and clinical parameters of $\mathrm{CP}$ and $\mathrm{HC}$ groups.

\begin{tabular}{|c|c|c|c|c|}
\hline \multirow{2}{*}{ Groups } & \multicolumn{2}{|c|}{ IL-17A $^{+}$IL-17F ${ }^{-}$Th17 } & \multicolumn{2}{|c|}{ IL-17A $^{-}$IL-17F $^{+}$Thl7 } \\
\hline & $\mathrm{HC}$ & $\mathrm{CP}$ & $\mathrm{HC}$ & $\mathrm{CP}$ \\
\hline Probing depth (PD) & 0.03 & $0.21^{*}$ & 0.04 & 0.01 \\
\hline Attachment loss (AL) & 0.08 & $0.17^{*}$ & 0.009 & $0.15^{*}$ \\
\hline Plaque index (PI) & 0.06 & 0.05 & 0.09 & 0.08 \\
\hline Gingival index (GI) & 0.10 & 0.02 & 0.03 & 0.11 \\
\hline
\end{tabular}

${ }^{*}$ Correlation is significant at $P<0.05$ level (Spearman correlation coefficient).

IL-17 production [26] and might be suppressed by molecular De-1 (developmental endothelial locus 1) [27], indicating the emerging role in periodontitis pathogenesis.

In vitro test showed that human recombinant IL-23 can significantly enhance the expression level of IL-17A $\mathrm{A}^{+} \mathrm{IL}-17 \mathrm{~F}^{-}$ and IL-17 $\mathrm{A}^{-}$IL-17 $\mathrm{F}^{+}$Th17 cells both in CP group and in HC group. However, to extent of our knowledge, precise role of IL-23 in local periodontal lesions is still obscure. In our previous study, we found the increased levels of IL-23 expression in diseased periodontal lesions, which suggested its involvement in the pathogenesis of periodontal destruction $[17,28]$. Previous reports showed that Th17 cells expressed IL-17 in an IL-23-dependent fashion [14]. Additionally, in our previous study, we did find significant elevated IL-23 expression level in local lesions in CP patients [18]. However, though serum IL-23 was not elevated significantly in CP, we still found an increased level of serum IL-17 in CP patients. This may be attributed to other Th17 promoting cytokines like IL-1 or IL-6 [14].

As previously reported, the periodontal diseases pathogenesis may not simply be accompanied by a consequence of Th1/Th2 balance, but these profiles of immune response may also be profoundly influenced by other subsets like Th17, which may play indispensable role in periodontitis. Together with conclusions of our previous studies, the present results have reconfirmed that the expression patterns of Th17 and its cytokines may be related to the clinical parameters of periodontitis and may be involved in the disease progression. As indicated above, chronic periodontitis demonstrated a chronic inflammation involved in the hazardous microbes infection and the consequent immuneinflammatory responses. Therefore, in the present study, we described the presence of three distinct Th17 cells in CP and HC groups. Apparently, the increased levels of IL-17A ${ }^{+}$IL$17 \mathrm{~F}^{-}$and IL-17 $\mathrm{A}^{-} \mathrm{IL}-17 \mathrm{~F}^{+}$Th17 cells in CP group correlated with attachment loss and probing depth, indicating there may be potential mechanism for these Th17 cells in periodontitis.

IL-17F recently has drawn much more attention due to its great similarity to IL-17A, and the genes encoding IL-17A and IL-17F are localized in the same chromosomal region and are coexpressed by Th17 cells [11, 12]. Similar to IL-17A, IL17F can also utilize IL-17RA and IL-17RC receptor complex as its signal transducers to induce the expression of proinflammatory cytokines in many human cell types [13]. In our 
present study, the flow cytometry results showed that there were significantly elevated levels of IL-17A $\mathrm{AL}^{+}-17 \mathrm{~F}^{-}$and IL$17 \mathrm{~A}^{-} \mathrm{IL}_{-17 \mathrm{~F}^{+}}$Th17 cells in CP group compared to HC group.

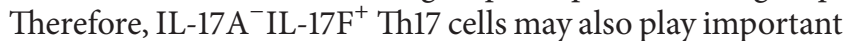
role in periodontal disease like IL-17 $\mathrm{A}^{+} \mathrm{IL}-17 \mathrm{~F}^{-}$Thl7 cell as both Th17 cells were correlated with the attachment loss in periodontitis. When under rhIL-23 stimulation, more IL$17 \mathrm{~A}^{+} \mathrm{IL}-17 \mathrm{~F}^{-}$Th17 cells would be produced in CP group than in $\mathrm{HC}$ group, and also only in $\mathrm{CP}$ group the change of IL$17 \mathrm{~A}^{-}$IL-17F ${ }^{+}$Th17 showed a significantly increase than in HC group. Therefore, the present study indicated to us that both IL-17A ${ }^{+}$IL- $17 F^{-}$and IL-17A $A^{-}$IL-17F ${ }^{+}$Th17 cells were totally involved in disease pathogenesis of CP group.

Taken together, this clinical study indicated that IL-17A ${ }^{+}$ and/or IL-17F $\mathrm{F}^{+}$positive Thl7 may participate in the periodontitis pathogenesis. Further epidemiological study between Th17 cells and periodontal diseases should be investigated, and possible pathological relationships should be further studied. Meanwhile, in further study, we should pay more attention to the role of Th17 associated cytokines IL-17A and IL-17F to test how these two cytokines affect the periodontal ligament fibroblast, through which signaling pathways the cytokine IL-17A and IL-17F may affect the pro-inflammatory mediator release.

\section{Conflict of Interests}

The authors declare that there is no conflict of interests regarding the publication of this paper.

\section{Acknowledgments}

This work was supported by Grants from the National Natural Science Foundation of China (81371150 and 81400517), China Postdoctoral Science Foundation funded Project (no. 2014M550767), and Project supported by Beijing Postdoctoral Research Foundation (2014ZZ002).

\section{References}

[1] Y.-T. A. Teng, "The role of acquired immunity and periodontal disease progression," Critical Reviews in Oral Biology and Medicine, vol. 14, no. 4, pp. 237-252, 2003.

[2] E. Gemmell, K. Yamazaki, and G. J. Seymour, "The role of T cells in periodontal disease: homeostasis and autoimmunity," Periodontology 2000, vol. 43, no. 1, pp. 14-40, 2007.

[3] S. Engebretson, R. Chertog, A. Nichols, J. Hey-Hadavi, R. Celenti, and J. Grbic, "Plasma levels of tumour necrosis factor$\alpha$ in patients with chronic periodontitis and type 2 diabetes," Journal of Clinical Periodontology, vol. 34, no. 1, pp. 18-24, 2007.

[4] P. M. Preshaw, A. L. Alba, D. Herrera et al., "Periodontitis and diabetes: a two-way relationship," Diabetologia, vol. 55, no. 1, pp. 21-31, 2012.

[5] S. Romagnani, E. Maggi, F. Liotta, L. Cosmi, and F. Annunziato, "Properties and origin of human Th17 cells," Molecular Immunology, vol. 47, no. 1, pp. 3-7, 2009.

[6] L. A. Tesmer, S. K. Lundy, S. Sarkar, and D. A. Fox, “Th17 cells in human disease," Immunological Reviews, vol. 223, no. 1, pp. 87-113, 2008.
[7] L. Steinman, "A brief history of $\mathrm{T}_{H} 17$, the first major revision in the $\mathrm{T}_{H} 1 / \mathrm{T}_{H} 2$ hypothesis of $\mathrm{T}$ cell-mediated tissue damage," Nature Medicine, vol. 13, no. 2, pp. 139-145, 2007.

[8] J. J. Yu, M. J. Ruddy, G. C. Wong et al., "An essential role for IL17 in preventing pathogen-initiated bone destruction: recruitment of neutrophils to inflamed bone requires IL-17 receptordependent signals," Blood, vol. 109, no. 9, pp. 3794-3802, 2007.

[9] S. L. Gaffen and G. Hajishengallis, "A new inflammatory cytokine on the block: re-thinking periodontal disease and the Th1/Th2 paradigm in the context of Th17 cells and IL-17," Journal of Dental Research, vol. 87, no. 9, pp. 817-828, 2008.

[10] N. M. Moutsopoulos, H. M. Kling, N. Angelov et al., "Porphyromonas gingivalis promotes Thl7 inducing pathways in chronic periodontitis," Journal of Autoimmunity, vol. 39, no. 4, pp. 294-303, 2012.

[11] S. H. Chang and C. Dong, "IL-17F: regulation, signaling and function in inflammation," Cytokine, vol. 46, no. 1, pp. 7-11, 2009.

[12] C. Dong, "Differentiation and function of pro-inflammatory Th17 cells," Microbes and Infection, vol. 11, no. 5, pp. 584-588, 2009.

[13] S. H. Chang and C. Dong, "A novel heterodimeric cytokine consisting of IL-17 and IL-17F regulates inflammatory responses," Cell Research, vol. 17, no. 5, pp. 435-440, 2007.

[14] K. Kreymborg, R. Etzensperger, L. Dumoutier et al., "IL-22 is expressed by Th17 cells in an IL-23-dependent fashion, but not required for the development of autoimmune encephalomyelitis," The Journal of Immunology, vol. 179, no. 12, pp. 8098-8104, 2007.

[15] M. J. McGeachy and D. J. Cua, "Th17 cell differentiation: the long and winding road," Immunity, vol. 28, no. 4, pp. 445-453, 2008.

[16] H. Ohyama, N. Kato-Kogoe, A. Kuhara et al., "The involvement of IL-23 and the Th 17 pathway in periodontitis," Journal of Dental Research, vol. 88, no. 7, pp. 633-638, 2009.

[17] H. Wang, Q. Han, Z. Luo et al., "Oral lichen planus may enhance the expression of Th17-associated cytokines in local lesions of chronic periodontitis," Clinical Oral Investigations, vol. 18, no. 6, pp. 1647-1654, 2014.

[18] H. Wang, Z. Luo, L. Lei et al., "Interaction between oral lichen planus and chronic periodontitis with Th17-associated cytokines in serum," Inflammation, vol. 36, no. 3, pp. 696-704, 2013.

[19] C. R. Cardoso, G. P. Garlet, G. E. Crippa et al., "Evidence of the presence of $\mathrm{T}$ helper type 17 cells in chronic lesions of human periodontal disease," Oral Microbiology and Immunology, vol. 24, no. 1, pp. 1-6, 2009.

[20] L. Zhao, Y. Zhou, Y. Xu, Y. Sun, L. Li, and W. Chen, "Effect of non-surgical periodontal therapy on the levels of Th17/Th1/Th2 cytokines and their transcription factors in Chinese chronic periodontitis patients," Journal of Clinical Periodontology, vol. 38, no. 6, pp. 509-516, 2011.

[21] E. Gemmell, K. Yamazaki, and G. J. Seymour, "Destructive periodontitis lesions are determined by the nature of the lymphocytic response," Critical Reviews in Oral Biology and Medicine, vol. 13, no. 1, pp. 17-34, 2002.

[22] E. Gemmell and G. J. Seymour, "Immunoregulatory control of Th1/Th2 cytokine profiles in periodontal disease," Periodontology 2000, vol. 35, pp. 21-41, 2004.

[23] T. Yago, Y. Nanke, N. Ichikawa et al., "IL-17 induces osteoclastogenesis from human monocytes alone in the absence of 
osteoblasts, which is potently inhibited by anti-TNF- $\alpha$ antibody: a novel mechanism of osteoclastogenesis by IL-17," Journal of Cellular Biochemistry, vol. 108, no. 4, pp. 947-955, 2009.

[24] C. Dong, "TH17 cells in development: an updated view of their molecular identity and genetic programming," Nature Reviews Immunology, vol. 8, no. 5, pp. 337-348, 2008.

[25] F. Sallusto and A. Lanzavecchia, "Heterogeneity of CD4 ${ }^{+}$memory T cells: functional modules for tailored immunity," European Journal of Immunology, vol. 39, no. 8, pp. 2076-2082, 2009.

[26] K. Sato, A. Suematsu, K. Okamoto et al., “Th17 functions as an osteoclastogenic helper $\mathrm{T}$ cell subset that links $\mathrm{T}$ cell activation and bone destruction," The Journal of Experimental Medicine, vol. 203, no. 12, pp. 2673-2682, 2006.

[27] S. A. Khader, "Restraining IL-17: Del-1 deals the blow," Nature Immunology, vol. 13, no. 5, pp. 433-435, 2012.

[28] Z. Luo, H. Wang, Z. Sun, W. Luo, and Y. Wu, "Expression of IL22, IL-22R and IL-23 in the peri-implant soft tissues of patients with peri-implantitis," Archives of Oral Biology, vol. 58, no. 5, pp. 523-529, 2013. 


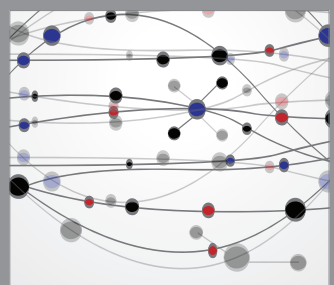

The Scientific World Journal
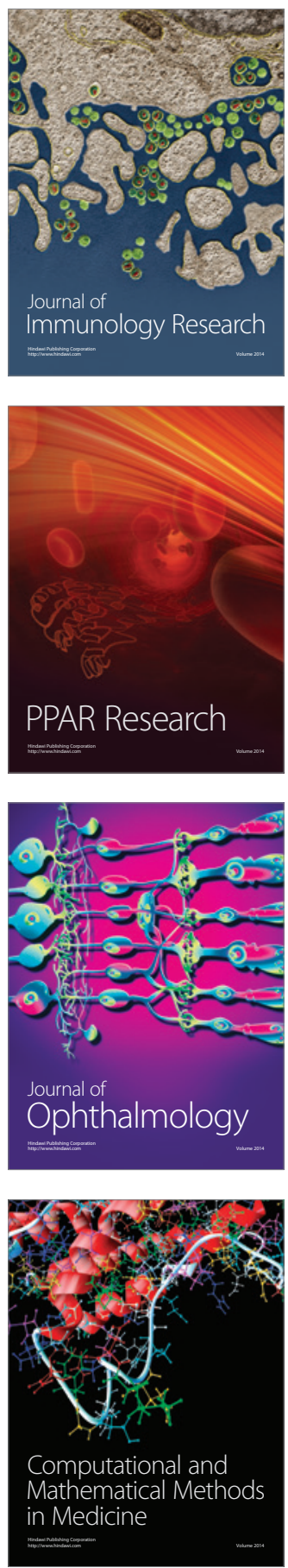

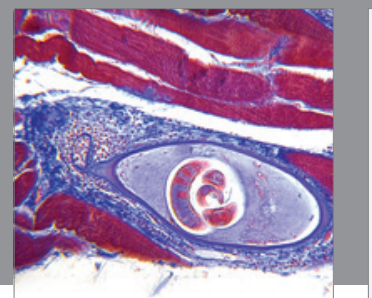

Gastroenterology

Research and Practice
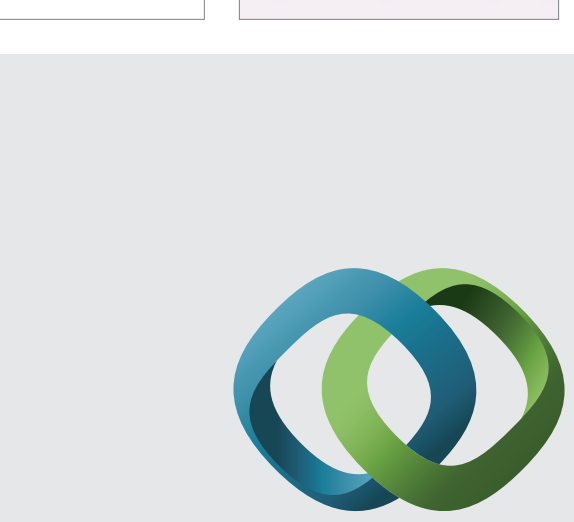

\section{Hindawi}

Submit your manuscripts at

http://www.hindawi.com
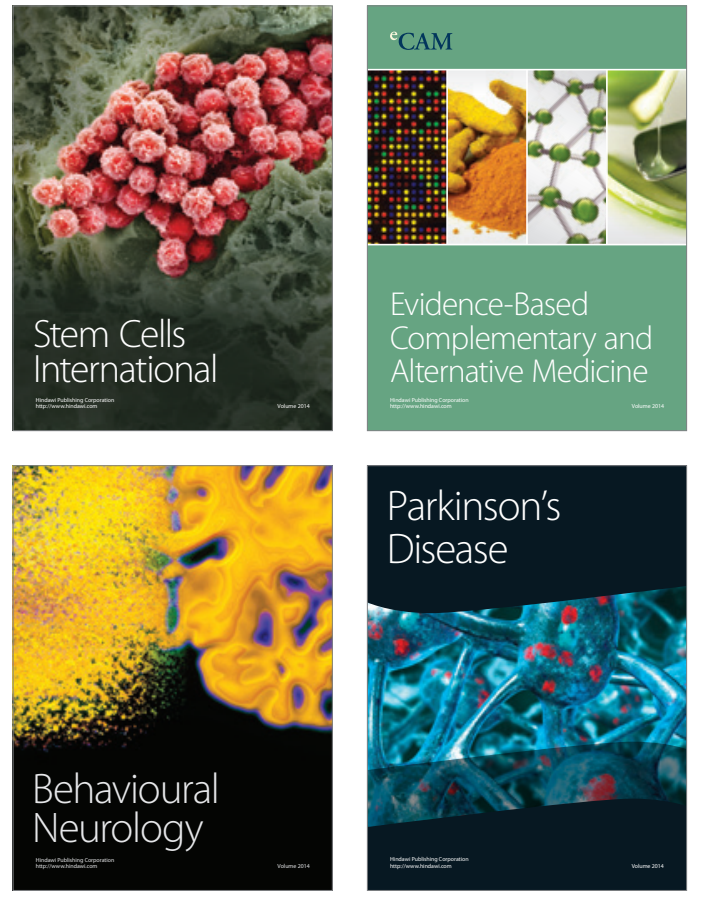
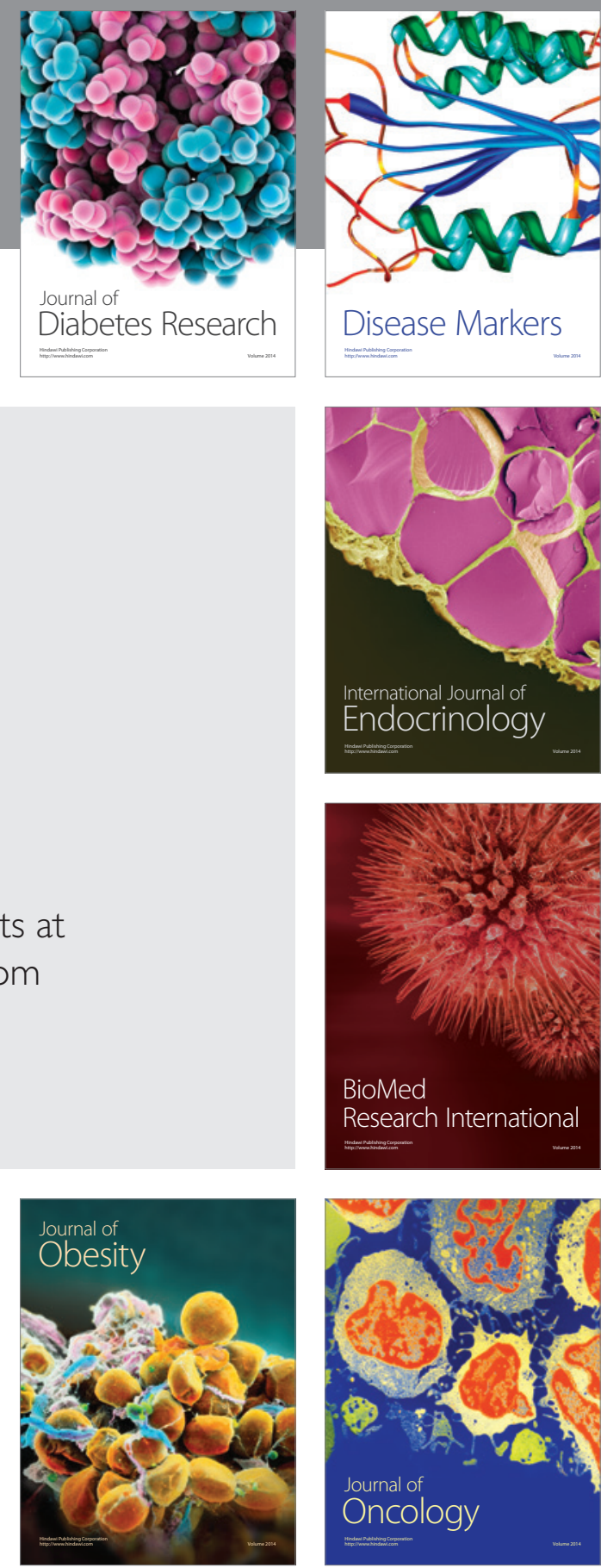

Disease Markers
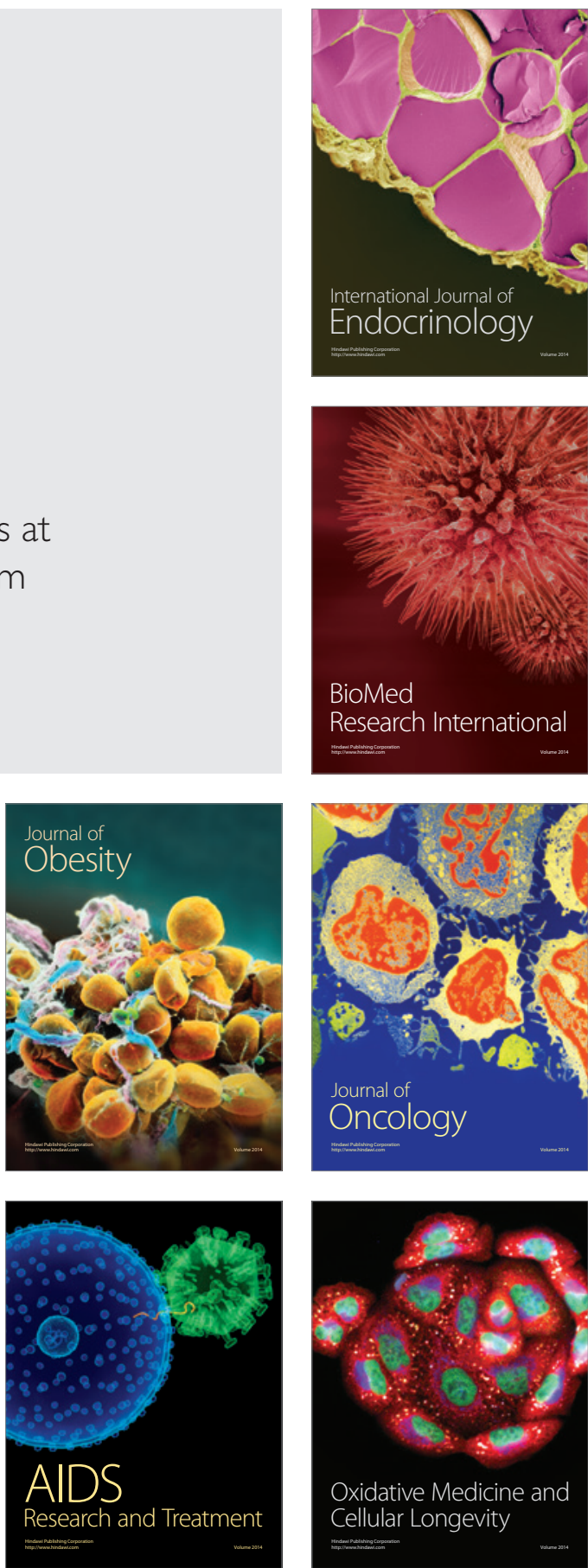